\title{
Financiamiento del sistema de pensiones mexicano por medio de bonos de longevidad Fernando Cruz-Aranda ${ }^{12}$ \\ Universidad Panamericana \\ Claudia Estrella Castillo Ramírez
}

Universidad Panamericana

Citlalli Pérez Flores

Universidad Panamericana

(Recibido 11 de septiembre 2017, aceptado 18 de diciembre 201\%.)

DOI: http://dx.doi.org/10.21919/remef.v13i3.303

\begin{abstract}
Resumen
El objetivo de este trabajo es valuar y analizar el bono de longevidad que representa una alternativa de inversión a largo plazo para fortalecer los fondos de pensiones de la población mexicana, hombres y mujeres, que alcanzará los 65 años. Esta presenta un incremento en la esperanza de vida, riesgo de longevidad, y una caída en la tasa de natalidad. Así, el valor del bono cupón cero es modelado a través de una ecuación que incorpora la tasa de mortalidad estimada a la edad x y al tiempo t, de cada género, con una proyección del índice de mortandad al 2040. Por medio del instrumento de deuda, se busca contrarrestar las pérdidas probables en las que incurriría una institución de seguros como consecuencia de la posible extensión de la vida esperada de los asegurados y bajar la probabilidad de incumplimiento del corporativo ante ellos y brindándoles mayor certidumbre; lo que coadyuva en dicho fondo al tener acceso al mercado de capitales y proporcione al retirado un mejor bienestar social.
\end{abstract}

Clasificación JEL: C01, C13, C65, G12, G23.

Palabras clave: Sistema de pensiones, envejecimiento poblacional, bono de longevidad.

\section{Financing of the Mexican pension system through longevity bonds}

\begin{abstract}
The objective of this work is to value and analyze the longevity bond that represents a long-term investment alternative to strengthen the pension funds of the Mexican population, that is, men and women who will reach 65 years of age. This presents an increase in life expectancy, risk of longevity, and a fall in the birth rate. Thus, the value of the zero-coupon bond is modeled through an equation that incorporates the estimated mortality rate at age $\mathrm{x}$ and time $\mathrm{t}$, of each gender, with a projection of the mortality rate for 2040. The debt instrument is used to try and offset the probable losses that would be incurred by an insurance institution as consequence of the possible extension of the expected life of the insured and lower the probability of default of the corporate before them and providing them greater certainty; which contributes to this

\footnotetext{
${ }^{1}$ Escuela de Ciencias Económicas y Empresariales, Universidad Panamericana, Campus México, México Augusto Rodin 498, México, D.F. 03920. Emails: fcruz@up.edu.mx; cecastillo@up.edu.mx; citlalli_08@icloud.com

${ }^{2}$ Persona que recibirá la correspondencia: Fernando Cruz Aranda, Tel.: Cel.: (55) 40130816; oficina: (55) 5482-1600; ext. 5477 correo: fcruz@up.edu.mx Dirección: Augusto Rodin 498, Col. Insurgentes Mixcoac, 03920, México, Ciudad de México.
} 
fund by providing access to the capital market and better social welfare to the retiree. JEL Classification: C01, C13, C65, G12, G23.

Keywords: Pension system; population aging; longevity bond.

\section{Introducción}

Una de las mayores preocupaciones sobre el fondo de pensiones es la administración del riesgo de longevidad, si la población supera la esperanza de vida esperada se presenta la posibilidad de que el fondo de pensiones no cubra a todos los pensionados. Por lo que un instrumento actuarial financiero denominado bono de longevidad podría cubrir o dar solución al problema de los pensionados.

El sistema público de pensiones en México, basados en el principio de reparto y sin una reforma adecuada en su estructura de financiamiento pone en riesgo la sustentabilidad de dicho sistemas de pensiones. Además, se observa que la población Mexicana está en una época de transición, es decir de una población joven a una población de edad avanzada.

El sistema reformado de México consiste en un plan de plena capitalización, con una garantía mínima y subvenciones para los afiliados de ingreso bajo. Los trabajadores que están a la mitad de su carrera pueden elegir entre el sistema anterior y el nuevo, y en el momento de su retiro tienen la posibilidad de optar entre los beneficios de un sistema o del otro. El sistema de pensiones (Centro de Estudios de las Finanzas Públicas, CEFP/008/ 2004) tiene como objetivo que los trabajadores cuenten al momento de su retiro con los recursos necesarios que les permitan alcanzar cierto nivel de consumo.

A nivel nacional la densidad de la población es de 61 habitantes por $\mathrm{Km}^{2}$ (Instituto Nacional de Estadística y Geografía, INEGI), siendo la Ciudad de México la de mayor densidad poblacional. La esperanza de vida que en 1970 se ubicaba en 61 años de edad, en 2015 está ubicada en aproximadamente 75 años de edad (INEGI), lo que significa un incremento en la esperanza de vida de alrededor del $18.67 \%$. Con este aumento en la esperanza de vida de la población Mexicana, el desempleo de adultos mayores, una población de edad avanzada que superará fuertemente a la población joven económicamente activa y que durante los siguientes dos o tres decenios dará lugar a un mayor deterioro en el sistema de pensiones actual y que rige desde el primero de julio de 1997(CEFP/008/ 2004), tomando en cuenta que aún está vigente el de 1973. El sistema de pensiones de 1997 sólo incluye a los trabajadores afiliados al Instituto Mexicano del Seguro Social, IMSS, y están excluidos el resto de los trabajadores. Los servicios de los que gozan los afiliados al IMSS es que la pensión, en caso de fallecimiento, será pagado por la aseguradora (régimen 1997) y será pagado por el IMSS para aquellos del régimen de 1973. Mientras que la pensión por cesantía en edad avanzada y vejez será pagada por IMSS, AFORE, Aseguradora para el régimen de 1997 y para el de 1973 sería pagada solo por el IMSS. Para el retiro por anticipado para los del régimen de 1973 no existe y para los de 1997 el pago es realizado por la aseguradora. Cualquier trabajador que haya cotizado hasta antes de 1997 puede elegir por el régimen que desee y dado que los trabajadores 
que estén en el régimen de 1973 tendrían que esperar a lo más 27 años y para algunos aún quedan alrededor de al menos tres años para que se jubilen bajo este régimen. Esto da lugar a una eminente crisis por un lado causada por el envejecimiento de la población y por el otro se pone en riesgo a las futuras generaciones que estarán asumiendo la carga que representan los adultos mayores y poniendo en riesgo las finanzas de las familias que desean un nivel de mejor calidad de vida, es decir tener un mecanismo o instrumento financiero que les permita garantizar suficiencia financiera.

Lo que ha conducido a que algunos trabajadores adopten un mecanismo de cuentas individuales, Vásquez C. (2012).

En México durante 1990, se tenía una tasa de natalidad de 27.91 por cada mil habitantes, para 2015 la tasa de natalidad fue de 18.52 por cada mil habitantes ${ }^{3}$. Además, la edad promedio de la población se ha incrementado, teniendo una mayor proporción de adultos de la tercera edad en el año 2050 y se espera que más de $16 \%$ de la población tenga una edad superior a los 65 años.

Esta transición demográfica representa retos en el financiamiento de servicios principalmente en salud y por supuesto en el pago de Pensiones. El riesgo de que una persona viva más años de los esperados (Esperanza de Vida), se le denomina Riesgo de Longevidad.

En 2005, la Organización para la Cooperación y Desarrollo Económico, OCDE; publicó el estudio: "El envejecimiento y la Reforma del Sistema de Pensiones", en el cual destaca que el principal desafío es encontrar la contraparte del mercado (inversionistas) que se beneficien con una mayor sobrevida de la población. Las industrias enfocadas en la tercera edad, como la industria farmacéutica, cumplen con este requerimiento, sin embargo esta oferta no sería suficiente para satisfacer la demanda por cobertura contra el Riesgo de Longevidad. Por lo tanto, también, se ha argumentado que el Gobierno podría ser el propulsor de este mercado. Por otra parte, los cambios demográficos y los cambios socio-económicos también tienen un impacto en los Sistemas de Pensiones. La caída de la natalidad, el aumento de la Esperanza de Vida en la vejez y la postergación de la edad de entrada al mercado laboral debido a la expansión de la cobertura educacional son factores que han reducido la proporción de trabajadores activos en comparación con la proporción de trabajadores pensionados. Es por esto que en los países con Sistemas de Reparto ${ }^{4}$, la sustentabilidad financiera es incierta.

\footnotetext{
${ }^{3}$ CONAPO. Indicadores Socio demográficos http://www.conapo.gob.mx/es/CONAPO/ Proyecciones_Datos

${ }^{4} \mathrm{El}$ sistema de reparto (o de beneficio definido por el monto de la pensión que recibe cada trabajador al llegar al retiro) está determinado por los años de servicio y por la edad, y no tiene relación con las contribuciones realizadas. Los trabajadores activos financian con sus contribuciones las pensiones de los trabajadores retirados. En contraparte, en un sistema de cuentas individuales o de contribución definida, los trabajadores activos tienen una cuenta individual donde acumulan sus contribuciones. Al momento del retiro, el monto de la pensión del trabajador está determinado por los fondos acumulados en su cuenta y los rendimientos generados a lo largo de su vida laboral.
} 
Una esperanza de vida mayor obliga a diversificar el riesgo de longevidad individual, que es posible solo si los mercados financieros ofrecen instrumentos adecuados para administrar este riesgo. Es de esta manera que se pueden tomar decisiones utilizando instrumentos de cobertura frente al riesgo.

Con la creciente presión financiera sobre los Planes de Pensiones, el Gobierno enfrenta múltiples desafíos a mediano y largo plazo. El costo del envejecimiento representa pasivos latentes para el gobierno, ya que están relacionados con la función del empleador y del proveedor de los servicios sociales.

Es probable que en las próximas décadas, la proporción del gasto público relacionado con el envejecimiento de la población tenga un incremento notable en relación con el Producto Interno Bruto (PIB). De no adoptarse nuevas reformas, se podrían generar presiones sobre las finanzas públicas y las calificaciones soberanas causadas por las reducciones del gasto en otros rubros o cambios en la distribución de riesgos, el costo financiero del envejecimiento y de los pasivos públicos que podrían incrementar la incertidumbre sobre las estimaciones y el alcance de esos pasivos.

Los administradores de los Fondos de Pensiones normalmente sostienen que se necesitan nuevos instrumentos financieros y una mayor oferta de instrumentos ya existentes para un mejor manejo de los riesgos de longevidad de la población y de la inflación. La longevidad representa un riesgo para las instituciones que administran estos productos debido a que si su tabla de mortalidad estimadas difieren fuertemente de los eventos reales implicaría el uso de mayores fondos para cubrir a los pensionados y esperar poder mantener la solvencia de la institución.

Estos instrumentos pueden ser complementados con la introducción de marcos normativos y una mayor orientación al mercado actual, considerando este tipo de riesgos de longevidad. Tales instrumentos incluyen los bonos de larga duración como los son: Bonos de Longevidad y los Bonos vinculados a la inflación. En muchos países se ha procurado desarrollar los mercados de estos bonos, sin embargo, la oferta sigue siendo pequeña en relación con la demanda potencial de los Fondos de Pensiones y de las compañías de seguros como lo describe Groome et. al. (Septiembre 2006).

Los Bonos de Longevidad son instrumentos financieros que asumen los costos de las pensiones de las personas que permanecen con vida en un periodo de tiempo determinado. Las entidades aseguradoras que administran coberturas al riesgo de supervivencia y saben del riesgo asociado a que el valor actuarial actual de los flujos de fondos futuros pudiera ser inferior al valor actual necesario para pagar las prestaciones en los términos previstos, como lo describe Ruiz F. (2016). Los Bonos de Longevidad podrían ser una muy buena alternativa del mercado de capitales para la cobertura del riesgo de longevidad como lo indican Bravo J. M. y Díaz G. J. (2014). 
Blake y Burrows (2001) consideran que el gobierno podría ayudar a los emisores de rentas vitalicias a cubrir el riesgo de la mortalidad agregada si introducen un nuevo tipo de vínculo, que denominan un bono de supervivencia. Los bonos de supervivencia permiten que la provisión de pensiones sea una responsabilidad compartida entre el público y sectores privados. Los cupones futuros del bono dependen del porcentaje de la población en edad de jubilación y que aún están vivos a la fecha del pago de cupones. Asimismo, Blake et. al. (2006) llevó a cabo una investigación en la cual evalúa el riesgo de longevidad a partir de la mortalidad agregada, utilizando derivados OTC (over the counter), permitiendo administrar el riesgo de longevidad. Wang J. (2008) realizó un trabajo de investigación con la población de Taiwán en el que se determina el precio de un Bono de Longevidad diferenciando a hombres y mujeres, en una fecha determinada de corte para la Esperanza de Vida.

Levantesi, Menziette y Torri (2008), realizaron un estudio para el mercado italiano a partir de anualidades utilizando la transformación de Wang (2002) y determinaron el precio del bono basado en un mercado libre de arbitraje utilizando derivados financieros. La transformación de Wang utilizada para ajustar la mejor estimación de la probabilidad de supervivencia. A continuación se describe dicha transformación.

Para ello, sea $X$ un cierto activo con función de densidad de probabilidad acumulada $F(x)$ y cuya función ajustada por riesgo, transformación de Wang, está dada por $g_{\lambda}(x)=\Phi\left[\Phi^{-1}(F(x))+\lambda\right], x \in(0,1)$, en la que $\Phi(x)$ es la función de distribución normal estándar y $\lambda$ es el parámetro de la transformada de Wang que representa el precio de mercado, esto es el nivel de riesgo sistemático. El valor del parámetro se determina de los precios de mercado de las rentas vitalicias.

El valor esperado ajustado por riesgo del rendimiento del activo i está dado por $E^{*}\left[R_{i}\right]=E\left[R_{i}\right]-\lambda \sigma_{i}$.

Por otro lado, la metodología de Miltersen y Persson (2005), en general estudia la evolución de la curva de la tasa de interés y la curva de la tasa forward instantánea y se ha utilizado en diferentes análisis para determinar el precio de los Bonos de Longevidad. En 2005 Miltersen y Persson realizaron un estudio con la metodología de Heath-Jarrow-Morton (HJM), y en 2006 con Cairns et. al. (2007) y Bauer D. (2006).

A lo largo de más de 15 años, se han propuesto diferentes metodologías para modelar el precio de los Bonos de Longevidad definiendo las probabilidades de supervivencia, comenzando en un punto de la curva para determinar el precio y la dinámica de los Bonos de Longevidad aplicando distintas metodologías aunque las más recurrentes han sido: la metodología Heath-Jarrow-Morton HJM; de acuerdo a Bauer D. y Ru J. (2006); donde retoman el trabajo hecho por Lin, Y. y Cox, S. (2005) y por Miltersen y Persson en (2005).

Otra alternativa que se muestra en las investigaciones es afrontar el riesgo de longevidad con un reaseguro muy similar al Credit Default Swap (CDS) utilizando la distribución de la transformación Wang. Por otra parte, los Bonos 
de Longevidad también han sido utilizados como una herramienta para transferir el riesgo de longevidad para el mercado de capitales bajo la estructura Credit Default Options (CDO); tal como lo propuso Liao, Yang y Huang en (2007). Otros trabajos que han tratado el tema de sistema de pensiones mexicano los puede ver en Maurillo-López, S. y Venegas-Martínez (2011) y Martínez-Preece, M. R. y Venegas-Martínez $(2014)^{6}$. En el presente documento y posterior a la introducción se describe en la sección dos el Bono de Longevidad y una descripción de la metodología de Lee y Carter (1992) y se considera que el modelo permite una adecuada descripción del comportamiento de la tasa de mortalidad al tiempo t y a la edad x y así analizar los bonos de longevidad como una alternativa de inversión a largo plazo para fortalecer los Fondos de Pensiones de la población Mexicana, hombres y mujeres, es decir a través de los bonos de longevidad contrarrestar las pérdidas probables en las que incurriría una institución de seguros como una consecuencia de la posible extensión de la esperanza de vida de los asegurados y evitar o disminuir la probabilidad de incumplimiento de la institución ante los asegurados y brindándoles mayor certidumbre. Por lo que se plantea la hipótesis: Los parámetros a estimar del modelo de Lee Carter permiten describir o explicar la tasa de mortalidad con un error por debajo del $2 \%$ en el periodo calculado para hombres y mujeres lo que implica o impacta en una alta bondad de ajuste y así poder realizar una valuación adecuada en los Bonos de longevidad para hombres y mujeres. En la sección tres se describen las características demográficas de la población mexicana y se determina una cohorte que se utilizará como base para modelar el comportamiento de la población y de su tasa de mortalidad. En la sección cuatro se considera la metodología de Lee y Carter en la que se estiman los parámetros del modelo de la tasa central de la mortalidad para hombres y mujeres, y se calcula el precio del Bono de Longevidad cupón cero en el periodo de 1990 a 2010 en la que se muestra la bondad de ajuste del modelo. Asimismo, se realizan proyecciones al 2040 para calcular el valor del Bono como función de la tasa de central de mortalidad al tiempo t y a la edad x para un periodo de 2018 al 2040 y además de los últimos 8 años al día de hoy (30 años) dada la información disponible por la secretaría de salud e INEGI. En la sección cinco se muestran las conclusiones sobre si un Bono de Longevidad representa una alternativa de inversión para los Fondos de Pensiones en la población mexicana, así como dar un conjunto de recomendaciones a nuevas líneas de investigación a futuro.

\section{Bono de longevidad y la metodología de Lee Carter}

A continuación se describen distintos bonos de longevidad y quienes estarían interesados en este tipo de bonos, así como la metodología de Lee Carter en la valuación de Bonos de longevidad.

\footnotetext{
${ }^{5}$ Cobertura de los sistemas de pensiones y factores asociados al acceso a una pensión de jubilación en México. Papeles de Población.

${ }^{6}$ Análisis del riesgo de mercado de los fondos de pensiones en México: un enfoque con modelos autorregresivos (2004-2010).
} 


\subsection{Bonos de Longevidad}

Un bono es un título de renta fija con fecha de vencimiento a corto o a largo plazo y paga dividendos, es decir cupones, de forma periódica y reembolsa el principal al vencimiento; y si no paga cupón se denomina Bono cupón cero es decir se realiza un solo pago. El Bono de longevidad, es un instrumento del mercado de deuda que proporciona al asegurador entrar al mercado de capitales y con riesgo subyacente del índice de mortandad y un riesgo agregado, en este último debido a los avances tecnológicos en la medicina y cuyo impacto es en el riesgo de longevidad. Los bonos sobre longevidad pagan altas rentabilidades pero los inversionistas están sujetos a la pérdida del total o de una parte del principal y de los intereses a lo largo de la vigencia del bono y proporcionando un nivel de certidumbre.

Un Bono de Longevidad es un instrumento de inversión definido por una función y pagos referenciados a un indicador o índice de mortandad de una población específica y se representa con un índice de supervivencia, La población inicial está basada en una cohorte determinada, por ejemplo los hombres de 65 años a mitad del año 2010 o bien a la edad x.

Existen distintos bonos de longevidad: i) Bonos de Longevidad clásicos.- A esta tipología corresponde la propuesta de Blake y Burrows (2001), con una emisión clásica de bonos cuyos cupones serían proporcionales a la tasa de supervivencia de una población específica y cuyo rembolso final se realizaría al fallecimiento del último superviviente de la cohorte de referencia. Por otro lado, en 2004 BNP Paribas (Líder europeo en servicios bancarios y financieros y considerado por la firma Standard Poors como uno de los seis bancos más sólidos del mundo) estructuro el primer bono de longevidad dirigido a planes de pensiones y a otros proveedores de rentas, y con una emisión de 540 millones y fecha de vencimiento a los 25 años (Leppisaari M., 2008). El Banco Europeo de Inversiones (EIB) colocaría en el mercado dichos bonos. Mientras que Partner RE fue la reaseguradora que asumió la cobertura del riesgo de longevidad. El tipo de bonos de longevidad abordados fue: ii) Bonos de Longevidad cupón cero.- Las emisiones de bonos cupón cero podrían ser diseñadas a partir de emisiones estándar de bonos de longevidad, con el objeto de facilitar el diseño de posiciones específicas de cobertura, con una doble perspectiva: una dimensión relacionada con el seguimiento de la cohorte y otra relacionada con la fecha de vencimiento; iii) Bonos de Longevidad diferidos.- Una de las críticas recibidas al bono EIB/BNP se refiere a que los cupones de los primeros años tienen un riesgo de longevidad reducido y en consecuencia, son flujos financieros relativamente caros. Para inversionistas cuyo objetivo del bono es el uso de instrumentos de cobertura, tales bonos utilizan grandes cuantías de capital para cubrir periodos de riesgo reducido. Una forma natural de resolver esta cuestión y hacer más atractivos los bonos para realizar coberturas sería crear emisiones de bonos con pagos diferidos. Los Bonos de Longevidad diferidos pueden ser considerados también como contratos forward de mortalidad; iv) Bonos de mortalidad con riesgo de principal.- Este bono tendría una estructura equivalente al bono Swiss 
Re, con pago anual de cupones y rembolso del principal al vencimiento. En circunstancias normales, que no superen el umbral establecido, los cupones y el principal se abonarían en su totalidad. Si el índice de mortalidad supera ciertos umbrales establecidos, se producen reducciones en el principal e incluso en los cupones periódicos. El índice de mortalidad se podría obtener como la media ponderada de diversos índices de mortandad, como se presenta en las opciones asiáticas.

\subsection{Interés de inversionistas y emisores por los Bonos de Longevidad}

A continuación se describe los posibles participantes a intervenir en un mercado de instrumentos de deuda y productos derivados bajo un activo subyacente de supervivencia o mortandad, por ejemplo: i) Las empresas de seguros.- Su objetivo es cubrirse de un determinado tipo de riesgo y en particular de la exposición al riesgo de longevidad, dentro de este riesgo están las empresas de seguros de vida y los proveedores de rentas vitalicias, que presentan exposiciones cruzadas, esto es, cuando se produce una disminución en la mortalidad los proveedores de rentas pierden mientras que las aseguradoras de vida ganan. Al compensarse las exposiciones da la oportunidad a una cobertura entre ambas carteras. Así, las compañías de seguros de vida con exposición al riesgo de longevidad y por arriba de su nivel de retención podrían contratar con una reaseguradora la cobertura del riesgo, como lo fue Partner RE, o transferirlo al mercado de capitales para dar mayor certidumbre; ii) Inversionistas.- Los bancos de inversión o aquellos que forman los fondos de cobertura y que estarían interesados en invertir en este tipo de instrumentos siempre y cuando tengan la posibilidad de obtener una buena rentabilidad y que les permita formar carteras diversificadas para minimizar el riesgo; iii) Especuladores y/o personas que realizan arbitrajes.En el mercado de derivados en el que se considera la mortalidad y que da la oportunidad de tener la participación de inversionistas a corto plazo que negocian activos acorde a los movimientos a la alza o a la baja de sus precios en el mercado. Éstos resultan útiles a la hora de disponer de liquidez, pues impactan en los mercados de instrumentos financieros como los futuros y las opciones de compra y/o venta del activo subyacente. Mientras que, las personas que realizan arbitrajes buscan obtener un beneficio de cualquier distorsión que reflejen los precios de los productos derivados y negociados en el mercado de valores; iv) Gobierno.- Podría incentivar la creación de estos mercados de derivados si presta apoyo a instituciones financieras expuestas al riesgo de longevidad, y así disminuir la probabilidad de quiebra o probabilidad de incumplimiento a través de los Fondos de Pensiones y obtener un mejor bienestar social acorde al entorno económico y financiero de los asegurados. Además, el gobierno podría administrar el riesgo de longevidad de los Fondos de Pensiones del sector privado y compañías aseguradoras y sería un incentivo para apoyar a las empresas de seguros a cubrir su exposición al riesgo de longevidad. 


\subsection{Modelo de Lee Carter}

En este documento de trabajo, la base del desarrollo de la proyección de la población mexicana de 65 años (hombres y mujeres) tendrán de base la metodología de Lee y Carter (1992) y es considerado como el punto de partida para la adopción del modelo cuya variable biométrica utilizada para la predicción es la tasa central de mortalidad con observaciones en el periodo de 1990 a 2010 para un intervalo de edades de $\mathrm{x}=0$ a 100 años. El modelo de Lee C. es un modelo valido en poblaciones no estacionarias. Una serie de tiempo $\left\{r_{t}\right\}$ es estrictamente estacionaria si la distribución conjunta de $\left(r_{t_{1}}, \ldots, r_{t_{k}}\right)$ es idéntica a la distribución conjunta de $\left(r_{t_{1}+t}, \ldots, r_{t_{k}+t}\right)$ para toda $\mathrm{t}$, donde $\mathrm{k}$ es un entero positivo y $\left(t_{1}, \ldots, t_{k}\right)$ es una colección de $\mathrm{k}$ enteros positivos, es decir ser estacionaria estricta requiere que la distribución conjunta $\left(r_{t_{1}}, \ldots, r_{t_{k}}\right)$ es invariante bajo el tiempo; para una mayor profundidad del tema de una población estacionaria, ver Tsay R. S. (2010).

El modelo de Lee Carter, es un modelo de la tasa de mortalidad (número de muertes en un rango específico para un tiempo determinado) de una población que alcanzara la edad $\mathrm{x}$ en un intervalo de tiempo t y cuyos parámetros pueden ser estimados en series de tiempo que permitirán realizar un análisis sobre el comportamiento futuro de dicha tasa y cuya propuesta original de estimación fue utilizar un modelo ARIMA. La parte fundamental del modelo Lee C. es la extrapolación de tendencias pasadas. Posteriormente otros modelos fueron desarrollados (Brouhns et. al., 2002; Renshaw y Haberman, 2003a, b; Currie et. al. 2004; Bongaarts, 2005; Girosi y King, 2006), pero el punto de referencia ha sido la metodología de Lee Carter el cual fue desarrollado para realizar pronósticos de largo plazo considerando series de tiempo históricas para distintas edades de la población de interés. También, se han planteado distintas modificaciones tales como utilizar funciones de densidad tales como la Binomial Negativas y Binomiales en lugar de Poisson y la estimación de parámetros en los modelos se puede realizar de distintas maneras: i) Descomposición singular de matrices a través de la función SVD (vea anexo A1), ii) Modelos lineales generalizados y iii) modelos no lineales generalizados.

En el modelo de Lee C. la evolución de las tasas de mortalidad que alcanzan la edad $\mathrm{x}$ al tiempo $\mathrm{t}$ sigue una dinámica de tipo exponencial a partir de un patrón promedio de la mortalidad en la cual las funciones biométricas dependen de la cohorte t.

La dinámica de la tasa de mortalidad está dada por

$$
m(t, x)=e^{a_{x}+b_{x} k_{t}+\epsilon_{x, t}}
$$

o bien en términos del logaritmo de $m(t, x)$, se tiene

$$
\log m(t, x)=a_{x}+b_{x} k_{t}+\epsilon_{x, t}
$$

En la que $\mathrm{x}=0,1,2, \ldots w_{t} ; t=t_{1}, t_{2}, t_{3}, \ldots, t_{n_{c}} ; \epsilon_{x, t} i . i . d . N\left(0, \sigma^{2}\right) . a_{x}, b_{x}, k_{t}$, son los parámetros a estimar en la que $w_{t}$ corresponde a la última edad a la que llegó la cohorte t, y en la que $k_{t}$ satisface una caminata aleatoria de la forma: 


$$
k_{t}=c+k_{t-1}+\xi_{t}
$$

Donde c es el cambio anual promedio del índice del nivel de mortalidad en el año t y dado por $k_{t} ; \epsilon_{t} \mathrm{y} \xi_{t}$ son vectores de ruido blanco.

El objetivo del Modelo de Lee-Carter es pronosticar una tasa de mortalidad en un período de $\mathrm{t}$ años de tiempo a la edad x; para ello se tiene que $a_{x}$ es el parámetro que determina el patrón de mortalidad de una edad específica, expresado como la media del logaritmo de la tasa de mortalidad $m(t, x)$ a la edad $\mathrm{x}$ en el tiempo $\mathrm{t} ; b_{x}$ es el parámetro que determina el cambio de la mortalidad a la edad $\mathrm{x}$ de acuerdo a los cambios en $k_{t}$ es decir

$$
\frac{d \log m(x, t)}{d t}=b_{x} \frac{d k_{t}}{d t}
$$

Así, $k_{t}$ es el índice de mortalidad o parámetro que define la tendencia de la mortalidad en el tiempo, es decir define la dirección de la esperanza de vida y ante una reducción en el índice de mortalidad habrá una mayor esperanza de vida; $\varepsilon_{x t}$ es el término de error, con media cero y varianza $\sigma_{\epsilon}^{2}$, y refleja influencias históricas que no son capturadas por el modelo.

El término $b_{x} k_{t}$ es bilineal y la estructura del modelo es invariante bajo transformaciones de los parámetros, es decir si $a_{x}, b_{x}$ y $k_{t}$ son una solución, entonces $\left(a_{x}-c b_{x}, b_{x}, k_{t}+c\right)$ son también solución $\forall c$, por lo que los parámetros han de ser normalizados y es tal que $\sum_{x} b_{x}^{i}=1$ y $\sum_{x} k_{x}^{i}=0$, con objeto de que el modelo tenga una única solución. El modelo no puede ser ajustado por las técnicas clásicas de regresión puesto que los valores índices $k_{t}$ no son observables, Brouhns Denuit (2002).

Sea $\mu_{x}$ la fuerza de la mortalidad y constante, es decir se garantiza uniformidad en la dinámica de mortalidad entre cada año, por lo que $\mu_{x+s, \tau}=\mu_{x, \tau}$, $0 \leq s<1$.

Por lo tanto,

$$
N_{x+1, \tau}=N_{x, \tau} e^{-\mu_{x, \tau}}=N_{x, \tau} e^{-e^{a_{x}+b_{x} k_{t}}}
$$

Ahora bien, para estimar los parámetros del modelo se utilizan mínimos cuadrados ordinarios, esto es

$$
\log \hat{m}(x, t)=\hat{a}_{x}+\hat{b}_{x} \bullet \hat{\kappa}_{t}+\hat{\epsilon}_{x, t}
$$

La condición de primer orden está dada por:

$$
\hat{a}_{x}=\frac{1}{T_{\text {end }}-T_{\text {begin }}+1} \sum_{t=T_{\text {begin }}}^{T_{\text {end }}} \log \hat{m}(x, t)
$$

Con $\hat{a}_{x}$ la media (en años) de la tasa de mortalidad. El estimador, se puede escribir como:

$$
\log \hat{m}(x, t)=\hat{a}_{x}+\underbrace{\hat{b}_{x} \bullet \hat{\kappa}_{t}} A_{x, t}+\hat{\epsilon}_{x, t}
$$

y utilizando los componentes de la descomposición de valor singular (vea apéndice A1) se obtienen los estimadores. 
La estimación de los parámetros en (5) se puede llevar a cabo mediante la descomposición en valores singulares (DVS) de la matriz:

$$
\log \left(\frac{q(x, t)}{1-q(x, t)}\right)-\hat{a}_{x}
$$

Los modelos lineales generalizado (GLM) condicionales como lo desarrolla Currie (2004) o el método de máxima verosimilitud, tal como propone Brouhns, Denuit y Vermut (2002), son también alternativas para determinar el valor de los parámetros.

El último paso del método de Lee-Carter consiste en ajustar una serie temporal a los valores de los índices de mortalidad $\left\{\hat{k}_{t}\right\}$. En muchas de las aplicaciones se obtiene un buen resultado con el modelo auto-regresivo de orden uno:

$$
\hat{k}_{t}=\alpha+\hat{k}_{t-1}+u_{t}
$$

con $\alpha$ constante y $u_{t}$ un ruido blanco.

La predicción para los años $t_{n}+s, s=1,2, \ldots$, posteriores al último $t_{n}$, se realiza sustituyendo en el modelo de Lee-Carter la predicción $\hat{k}_{t_{n+s}}$ obtenida a partir de la serie temporal ajustada,

$$
\log \left(\frac{\hat{q}_{x, t_{n+s}}}{1-\hat{q}_{x, t_{n+s}}}\right)=a_{x}+\hat{b}_{x} \hat{k}_{t_{n+s}}, \quad s>0
$$

Un método alternativo de predicción es el propuesto en Lee (2000), Renshaw y Haberman (2003b) haciendo uso de los últimos datos como punto de partida. Motivados por la necesidad de pronosticar factores actuariales de reducción de la mortalidad y trabajando dentro de un marco de modelado lineal generalizado, proponen una familia de predictores lineales cuya estructura es similar a la del modelo Lee C. Esta familia automáticamente asegura que el total de muertes son idénticos en cada año y es lo suficientemente flexible para permitir características específicas diferenciales de la edad de la persona. Entonces la tasa de mortalidad proyectada (Renshaw y Haberman, 2006) estará dada por

$$
\log \left(\frac{\hat{q}_{x, t_{n+s}}}{1-\hat{q}_{x, t_{n+s}}}\right)=\log \left(\frac{\dot{q}_{x t_{n}}}{1-\dot{q}_{x t_{n}}}\right)+\hat{b}_{x}\left(\hat{k}_{t_{n+s}}-\hat{k}_{t_{n+s}}\right), s>0
$$

Algunos autores han aportado sugerencias al método, entre ellos Wilmoth (1993) que proponen modificaciones al método de Lee-Carter con el fin de poder predecir la mortalidad para países que forman parte de un grupo, en lugar de considerarlos individualmente.

La principal crítica al modelo de Lee-Carter es que los parámetros $a_{x}$ y $b_{x}$ dependen sólo de la edad y que la predicción de futuros valores de la mortalidad se basa sólo en $k_{t}$, lo que supone admitir que no existe interacción entre la edad y el tiempo. Sus ventajas son, entre otras, la fácil interpretación de sus parámetros y su parsimonia. El modelo tiene actualmente mucha popularidad debido a sus buenos resultados y a su simplicidad.

Adicionalmente, se ha establecido empíricamente que el término dependiente del tiempo $k_{t}$, a menudo es lineal en la mayor parte del período de observación. 
Esta es una característica útil ya que permite una interpretación y proyección relativamente fácil.

El vector a se puede interpretar como un perfil de edad media, el vector $\mathrm{k}$ de seguimiento de los cambios de mortalidad en el tiempo y el vector b indica la cantidad de cada grupo de edad de los cambios cuando $k_{t}$ cambia. Cuando $k_{t}$ es lineal en el tiempo de cada grupo de edad cambia en su propio ritmo exponencial, pero no es un requisito del modelo. El término de error refleja el periodo de edad de los efectos no reflejados en el modelo.

\subsection{El valor del Bono cupón cero}

A continuación se describe la ecuación para determinar el valor del Bono que estará en función del plazo T-t, y de la dinámica de la tasa de mortalidad $m(t, x)$ y en la que el $\log a r i t m o$ de la tasa de mortalidad es $\log m(t, x)=a_{x}+b_{x} k_{t}+\epsilon_{x, t}$, siendo $x=0,1,2, \ldots w_{t} ; t=t_{1}, t_{2}, t_{3}, \ldots, t_{n_{c}} ; \epsilon_{x, t} \sim i . i . d . N\left(0, \sigma^{2}\right)$. Donde $a_{x}, b_{x}, k_{t}$, son los parámetros a estimar en la que $w_{t}$ corresponde a la última edad a la que llegó la cohorte t, y en la que $k_{t}$ satisface una caminata aleatoria, ecuación (3). Así el valor del bono está dado por

$$
B\left(m\left(t_{i}, x\right), T-t_{i}\right)=e^{-m\left(t_{i}, x\right)\left(T-t_{i}\right)} ; \quad i=1,2, \ldots, n_{c}
$$

\section{Características demográficas de la población mexicana}

A continuación, se presentan las características demográficas de hombres y mujeres de la población Mexicana en el periodo de 1990 a 2010. Las gráficas y estimación de parámetros se realizaron en el software MATLAB $^{\circledR}$ e Eviews ${ }^{\circledR}$ así como algunas gráficas en Excel $^{\circledR}$.

En la gráfica 1 se puede observar que la mortalidad del periodo de 1990 al año 2010 y considerando edades que van de $x=0$ hasta $x=100$ años de edad que ha alcanzado la persona ha ido disminuyendo notablemente la mortalidad y sobre todo en su primer año de vida, seguro social.

Gráfica 1. Mortalidad de la Población de hombres a la edad x en el año calendario t.

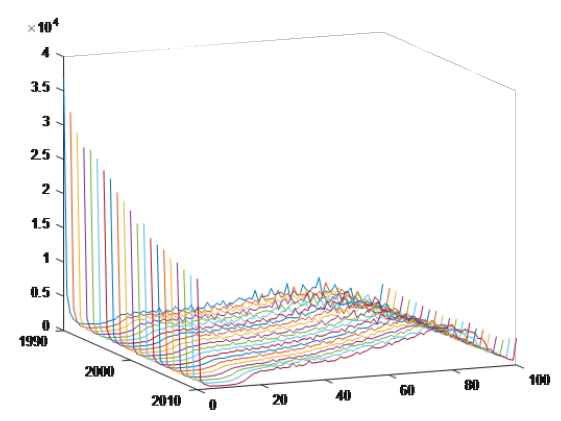

Fuente: Elaboración propia. Datos de INEGI y Secretaría de Salud. 
Mientras que en la gráfica 2 se puede observar que la tasa central de mortalidad de hombres está muy cercana a $1.13 \%$ a los 60 años y se empieza a incrementar suavemente con una volatilidad de $0.0886 \%$ como se describe en el cuadro 1, es decir la población empieza a presentar longevidad lo que implica un riesgo para las aseguradoras ya que las personas están presentando una esperanza de vida mayor que lo esperado.

Gráfica 2. Tasa central de mortalidad de la población de hombres a la edad x, en el año calendario t.

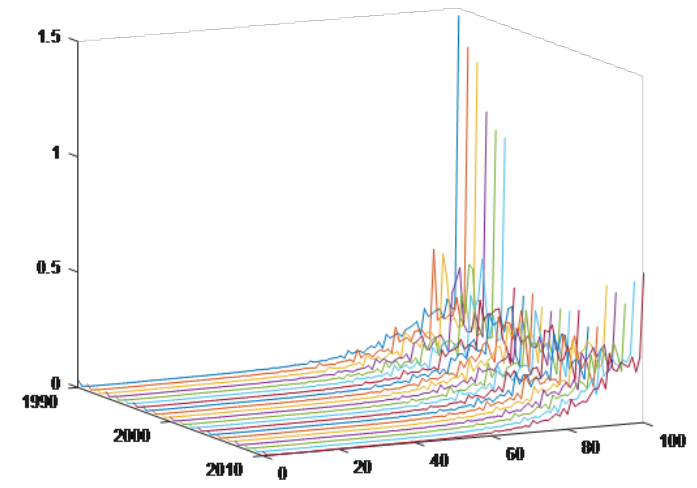

Fuente: Elaboración propia. Datos de INEGI y Secretaría de Salud.

Cuadro 1. Mortalidad de hombres en promedio y su volatilidad mínima y máxima en el periodo de 1990 a 2010

\section{Mortalidad en Hombres}

\begin{tabular}{|l|c|c|c|c|c|c|}
\hline & \multicolumn{3}{|c|}{ Tasa promedio } & \multicolumn{3}{c|}{ Volatilidad } \\
\hline & Minimo & & Máximo & Minimo & & Máximo \\
\hline & $0.03 \%$ & $1.13 \%$ & $64.70 \%$ & $0.01 \%$ & $0.09 \%$ & $38.54 \%$ \\
\hline Edad & 10 & 60 & 100 & 13 & 60 & 100 \\
\hline & & & & & & \\
\hline
\end{tabular}

Fuente: Elaboración propia. Datos de INEGI y Secretaría de Salud. 
400 Nueva Época REMEF (The Mexican Journal of Economics and Finance)

En la gráfica 3 se observa que la mortalidad del periodo de 1990 al año 2010 ha ido disminuyendo notablemente y de manera muy clara entre los niños que están por alcanzar su primer año de vida. A una edad de alrededor de los 70 años se dispara el número de muertes y empieza a decrecer lo que indica que existe un cierto número de personas longevas.

Gráfica 3. Mortalidad de la población de mujeres a la edad x en el año calendario t.

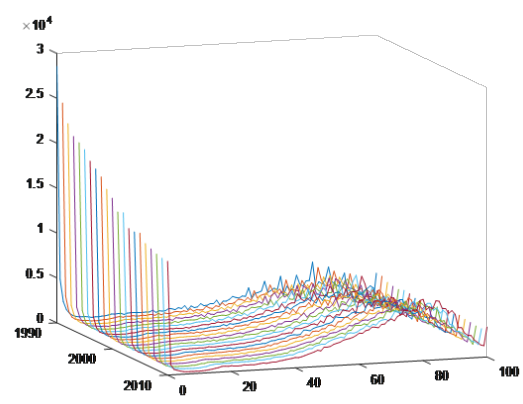

Fuente: Elaboración propia. Datos de INEGI y Secretaría de Salud.

En la gráfica 4 se puede observar que en el periodo de 1990 a 2010 la tasa promedio de mortalidad de mujeres es de $0.741 \%$ a los 60 años y se empieza a incrementar suavemente con una volatilidad que va de $0.003 \%$ hasta el $32 \%$, es decir la población de mujeres empieza a presentar longevidad lo que implica un riesgo para las aseguradoras ya que las personas están presentando una esperanza de vida mayor que lo esperado.

Gráfica 4. Tasa central de mortalidad de la población de mujeres a la edad x, en el año calendario t.

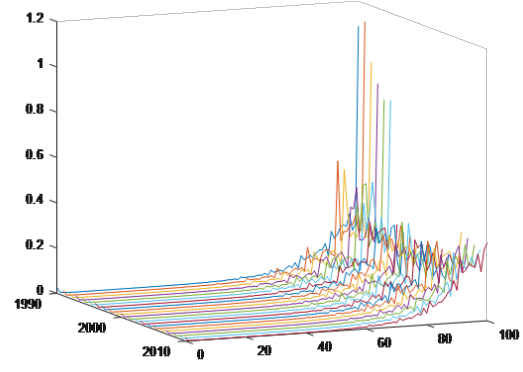

Fuente: Elaboración propia. Datos de INEGI y Secretaría de Salud. 
A continuación se presenta la tasa de mortalidad de mujeres.

Cuadro 2. Mortalidad de mujeres en promedio y su volatilidad mínima y máxima en el periodo de 1990 a 2010.

\begin{tabular}{|l|l|l|l|l|l|}
\hline & \multicolumn{5}{|c|}{ Mortalidad en Mujeres } \\
\hline & \multicolumn{4}{|c|}{ Tasa promedio } & Volatilidad promedio \\
\hline & Minimo & & Máximo & Minimo & Máximo \\
\hline & $0.03 \%$ & $0.05 \%$ & $46.19 \%$ & $0.00 \%$ & $32.09 \%$ \\
\hline Edad & 10 & 60 & 100 & 15 & 100 \\
\hline
\end{tabular}

Fuente: Elaboración propia. Datos de INEGI y Secretaría de Salud.

\section{Estimaciones de los parámetros del modelo Lee Carter y el valor del Bono cupón cero}

A continuación se presenta los valores obtenidos de las estimaciones del Modelo Lee Carter y el valor del Bono cupón cero para hombres y mujeres.

\subsection{Parámetros y el valor del Bono para hombres}

En el cuadro 3, 4 y 5 se muestran las estimaciones de los parámetros, $a_{x}, b_{x}$, y $k_{t}$, utilizando descomposición en valores singulares, DVS (apéndice A1), (Shores, T., 2004) del modelo de Lee Carter con base en los datos del cálculo de la tasa de mortalidad, $m(x, t)$, para hombres.

Cuadro 3. Parámetro $\hat{a}(x)$ a la edad $\mathrm{x}$.

\begin{tabular}{|cc|cc|cc|cc|cc|}
\hline$x$ & $\hat{a}(x)$ & $x$ & $\hat{a}(x)$ & $x$ & $\hat{a}(x)$ & $x$ & $\hat{a}(x)$ & $x$ & $\hat{a}(x)$ \\
\hline 0 & -3.8694 & & & & & & & & \\
1 & -6.2142 & 21 & -6.3081 & 41 & -5.3198 & 61 & -3.7161 & 81 & -2.024 \\
2 & -6.9359 & 22 & -6.3306 & 42 & -5.5948 & 62 & -3.9815 & 82 & -2.2751 \\
3 & -7.41 & 23 & -6.2928 & 43 & -5.3965 & 63 & -3.936 & 83 & -2.1344 \\
4 & -7.6809 & 24 & -6.2572 & 44 & -5.2734 & 64 & -3.8436 & 84 & -2.1244 \\
5 & -7.7744 & 25 & -6.201 & 45 & -5.3422 & 65 & -3.954 & 85 & -2.3087 \\
\hline
\end{tabular}


402 Nueva Época REMEF (The Mexican Journal of Economics and Finance)

\begin{tabular}{|cc|cc|cc|cc|cc|}
\hline$x$ & $\hat{a}(x)$ & $x$ & $\hat{a}(x)$ & $x$ & $\hat{a}(x)$ & $x$ & $\hat{a}(x)$ & $x$ & $\hat{a}(x)$ \\
\hline 6 & -7.8748 & 26 & -6.166 & 46 & -5.1544 & 66 & -3.6445 & 86 & -2.0496 \\
7 & -7.9214 & 27 & -6.1546 & 47 & -5.0614 & 67 & -3.5787 & 87 & -1.9677 \\
8 & -7.9657 & 28 & -6.1015 & 48 & -5.1196 & 68 & -3.5702 & 88 & -1.9228 \\
9 & -7.9659 & 29 & -6.0815 & 49 & -5.0573 & 69 & -3.3845 & 89 & -1.9355 \\
10 & -7.9959 & 30 & -6.0994 & 50 & -5.1374 & 70 & -3.7361 & 90 & -2.2665 \\
11 & -7.882 & 31 & -5.8715 & 51 & -4.6044 & 71 & -2.8273 & 91 & -1.1468 \\
12 & -7.8375 & 32 & -5.9909 & 52 & -4.8375 & 72 & -3.2058 & 92 & -1.4208 \\
13 & -7.6472 & 33 & -5.9152 & 53 & -4.6864 & 73 & -3.0146 & 93 & -1.355 \\
14 & -7.4301 & 34 & -5.8686 & 54 & -4.6434 & 74 & -2.9806 & 94 & -1.3437 \\
15 & -7.2635 & 35 & -5.7477 & 55 & -4.6363 & 75 & -3.1556 & 95 & -1.5943 \\
16 & -6.954 & 36 & -5.7829 & 56 & -4.5297 & 76 & -2.8686 & 96 & -1.5918 \\
17 & -6.7717 & 37 & -5.6748 & 57 & -4.3167 & 77 & -2.7032 & 97 & -1.4111 \\
18 & -6.6269 & 38 & -5.7179 & 58 & -4.3717 & 78 & -2.8455 & 98 & -1.7971 \\
19 & -6.4135 & 39 & -5.6774 & 59 & -4.2714 & 79 & -2.5783 & 99 & -2.13 \\
20 & -6.4673 & 40 & -5.7235 & 60 & -4.4872 & 80 & -2.9684 & 100 & -0.5825 \\
\hline
\end{tabular}

Fuente: Elaboración propia. De la ecuación (2) y datos de la Secretaría de Salud e INEGI.

Cuadro 4. Parámetro $\hat{b}(x)$ a la edad $\mathrm{x}$.

\begin{tabular}{|cc|cc|cc|cc|cc|}
\hline$x$ & $\hat{b}(x)$ & $x$ & $\hat{b}(x)$ & $x$ & $\hat{b}(x)$ & $x$ & $\hat{b}(x)$ & $x$ & $\hat{b}(x)$ \\
\hline 0 & 0.0229 & & & & & & & & \\
1 & 0.0378 & 21 & 0.0129 & 41 & 0.013 & 61 & 0.0104 & 81 & -0.0023 \\
2 & 0.0318 & 22 & 0.0175 & 42 & 0.0177 & 62 & 0.0118 & 82 & 0.003 \\
3 & 0.0258 & 23 & 0.0137 & 43 & 0.0118 & 63 & 0.0119 & 83 & -0.0018 \\
4 & 0.0239 & 24 & 0.0132 & 44 & 0.0135 & 64 & 0.0089 & 84 & 0.0001 \\
5 & 0.024 & 25 & 0.0141 & 45 & 0.0158 & 65 & 0.0054 & 85 & -0.005 \\
6 & 0.0247 & 26 & 0.0132 & 46 & 0.0124 & 66 & 0.0034 & 86 & 0.0005 \\
7 & 0.0228 & 27 & 0.0124 & 47 & 0.014 & 67 & 0.0052 & 87 & 0.001 \\
8 & 0.0226 & 28 & 0.0144 & 48 & 0.013 & 68 & 0.0068 & 88 & -0.0011 \\
9 & 0.0206 & 29 & 0.0075 & 49 & 0.0107 & 69 & 0.0084 & 89 & -0.0099 \\
10 & 0.0204 & 30 & 0.0146 & 50 & 0.0095 & 70 & -0.0006 & 90 & -0.0168 \\
\hline
\end{tabular}




\begin{tabular}{|cc|cc|cc|cc|cc|}
\hline$x$ & $\hat{b}(x)$ & $x$ & $\hat{b}(x)$ & $x$ & $\hat{b}(x)$ & $x$ & $\hat{b}(x)$ & $x$ & $\hat{b}(x)$ \\
\hline 11 & 0.017 & 31 & 0.0102 & 51 & 0.0109 & 71 & 0.0084 & 91 & 0.0049 \\
12 & 0.014 & 32 & 0.0155 & 52 & 0.0112 & 72 & 0.0076 & 92 & 0.0018 \\
13 & 0.0106 & 33 & 0.0135 & 53 & 0.0065 & 73 & 0.0088 & 93 & -0.0012 \\
14 & 0.0114 & 34 & 0.013 & 54 & 0.0075 & 74 & 0.0073 & 94 & -0.0066 \\
15 & 0.0074 & 35 & 0.0177 & 55 & 0.0088 & 75 & -0.0002 & 95 & -0.0054 \\
16 & 0.0071 & 36 & 0.0175 & 56 & 0.0079 & 76 & -0.001 & 96 & -0.013 \\
17 & 0.0122 & 37 & 0.0171 & 57 & 0.0077 & 77 & -0.0011 & 97 & 0.0014 \\
18 & 0.0147 & 38 & 0.0194 & 58 & 0.0082 & 78 & -0.0002 & 98 & -0.0033 \\
19 & 0.0152 & 39 & 0.0128 & 59 & 0.0078 & 79 & -0.0038 & 99 & 0.0103 \\
20 & 0.0126 & 40 & 0.0172 & 60 & 0.0055 & 80 & -0.0109 & 100 & 0.0568 \\
\hline
\end{tabular}

Fuente: Elaboración propia. De la ecuación (2) y datos de la Secretaría de Salud e INEGI.

Cuadro 5. Parámetro $\hat{k}(t)$ al tiempo t.

\begin{tabular}{|ccc|ccc|}
\hline$t$ & fecha & $\hat{k}(t)$ & $t$ & fecha & $\hat{k}(t)$ \\
\hline 0 & 1990 & 17.7932 & & & \\
1 & 1991 & 14.0059 & 11 & 2001 & -6.7784 \\
2 & 1992 & 12.1353 & 12 & 2002 & -6.2215 \\
3 & 1993 & 10.8018 & 13 & 2003 & -6.9594 \\
4 & 1994 & 8.4553 & 14 & 2004 & -8.702 \\
5 & 1995 & 7.3655 & 15 & 2005 & -8.0707 \\
6 & 1996 & 1.3886 & 16 & 2006 & -7.8497 \\
7 & 1997 & 0.3223 & 17 & 2007 & -7.4118 \\
8 & 1998 & -0.4196 & 18 & 2008 & -6.0167 \\
9 & 1999 & -3.2797 & 19 & 2009 & -2.3957 \\
10 & 2000 & -5.8127 & 20 & 2010 & -2.35 \\
\hline
\end{tabular}

Fuente: Elaboración propia. De la ecuación (3) y (7) y datos de la Secretaría de Salud e INEGI.

Tomando los valores estimados de $k_{t}$ y considerando un auto-regresivo de orden uno, AR(1), es decir tomando la ecuación (7), se tiene que

$$
\hat{k}_{t}=0,981447 \hat{k}_{t-1}
$$


404 Nueva Época REMEF (The Mexican Journal of Economics and Finance)

Por lo que la predicción para los años $t_{n}+s, s=1,2, \ldots, 30$ posteriores al último $t_{n}$, se realiza sustituyendo en el modelo de Lee-Carter la predicción $\hat{k}_{t_{n+s}}$ obtenida a partir de la serie temporal ajustada.

Gráfica 5. La descripción de $k_{t}$ y su autorregresivo de orden uno, 1990-2010

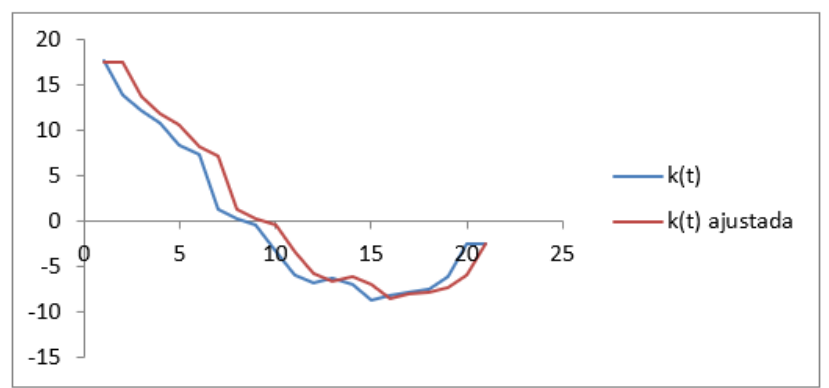

Fuente: Elaboración propia. De la ecuación (3) y (7) y datos de la Secretaría de Salud e INEGI.

En el cuadro 6 se presenta el estadístico de la bondad de ajuste, $R^{2}$, que indica el porcentaje que es capaz de describir el modelo del comportamiento de los datos observados de la tasa de mortandad, $m(x, t)$, para hombres y los estimados $\hat{m}(x, t)$ con el modelo de Lee Carter. Es decir, la bondad de ajuste considero la razón de la suma del cuadrado de los errores de las observaciones y el ajuste con el modelo y la suma del cuadrado de los errores de las observaciones y la media de las observaciones.

Cuadro 6. Bondad de ajuste de la tasa de mortandad $m(x, t)$ y $\hat{m}(x, t)$.

\begin{tabular}{|ccc|ccc|c|}
\hline & & & & & & $R^{2}(t)$ \\
$t$ & Año & $R^{2}(t)$ & $t$ & Año & $R^{2}(t)$ & Promedio \\
\hline 0 & 1990 & $99.50 \%$ & & & & $99.74 \%$ \\
1 & 1991 & $99.78 \%$ & 11 & 2001 & $99.84 \%$ & \\
2 & 1992 & $99.72 \%$ & 12 & 2002 & $99.81 \%$ & \\
3 & 1993 & $99.70 \%$ & 13 & 2003 & $99.84 \%$ & \\
4 & 1994 & $99.60 \%$ & 14 & 2004 & $99.84 \%$ & \\
5 & 1995 & $99.67 \%$ & 15 & 2005 & $99.85 \%$ & \\
6 & 1996 & $99.41 \%$ & 16 & 2006 & $99.74 \%$ & \\
7 & 1997 & $99.53 \%$ & 17 & 2007 & $99.79 \%$ & \\
8 & 1998 & $99.63 \%$ & 18 & 2008 & $99.76 \%$ & \\
9 & 1999 & $99.87 \%$ & 19 & 2009 & $99.92 \%$ & \\
10 & 2000 & $99.90 \%$ & 20 & 2010 & $99.89 \%$ & \\
\hline
\end{tabular}

Fuente: Elaboración propia. 
Se grafica el logaritmo natural de la tasas de mortalidad de datos históricos en el año 2010 y los estimados con el modelo de Lee Carter para el año 2010 con objeto de visualizar el ajuste en un año y en el que se observa del cuadro 6 que tiene una bondad de ajuste de $R^{2}=99,89 \%$.

Gráfica 6. Logaritmo natural de la Tasa de mortalidad del histórico y estimado para hombres

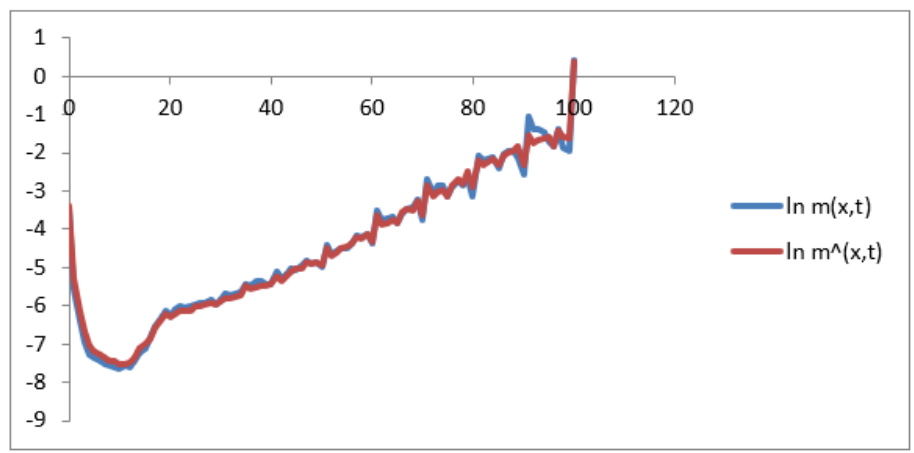

Fuente: Elaboración propia. De la ecuación (5) y los datos de la Secretaría de Salud e INEGI.

En la gráfica 7 se presenta el logaritmo natural de la mortalidad y en la cual se utilizó la ecuación (2) en la que se ha estimado los parámetros $a_{x}, b_{x}, \mathrm{y} k_{t}$, para los años de 1990 al 2010.

Gráfica 7. De la ecuación (2) en la que se estimaron los parámetros $a_{x}, b_{x}, \mathrm{y} k_{t}$.

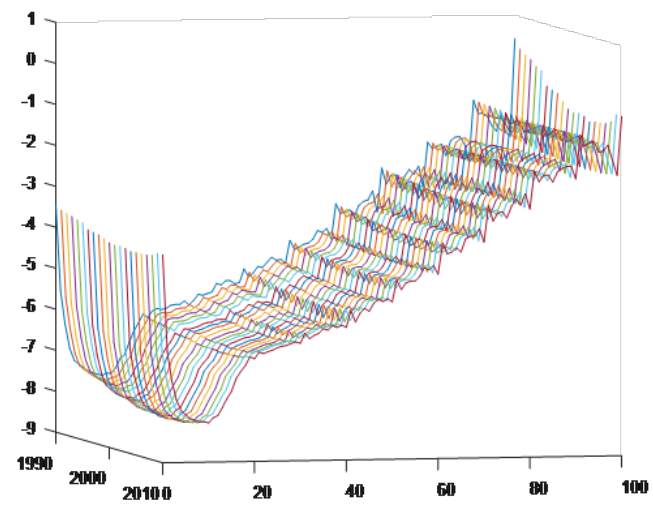

Fuente: Elaboración propia. De la ecuación (5) y los datos de la Secretaría de Salud e INEGI. 
406 Nueva Época REMEF (The Mexican Journal of Economics and Finance)

Gráfica 8. Dinámica de la tasa de mortalidad, m(t,x), de la población de hombres a la edad x, en el año calendario t.

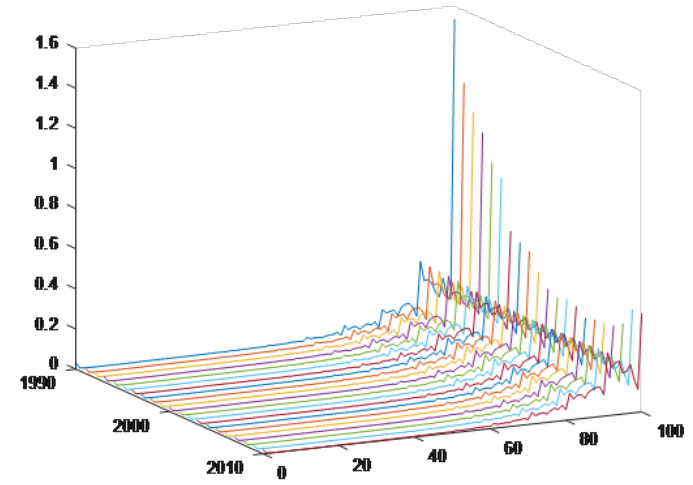

Fuente: Elaboración propia. Datos de la Secretaría de Salud e INEGI.

\subsubsection{El cálculo del Bono tomando $\hat{m}(x, t)$.}

Considerando la ecuación (8), (9) y (10) se calculó el valor del bono con la tasa $\hat{m}(x, t)$ estimada para hombres en el período de 1990 al 2010, y se grafica el valor del bono en función del año t y la edad x, gráfica 9.

Gráfica 9. Valor estimado del Bono que considera una tasa de mortandad de hombres a la edad $\mathrm{x}$.

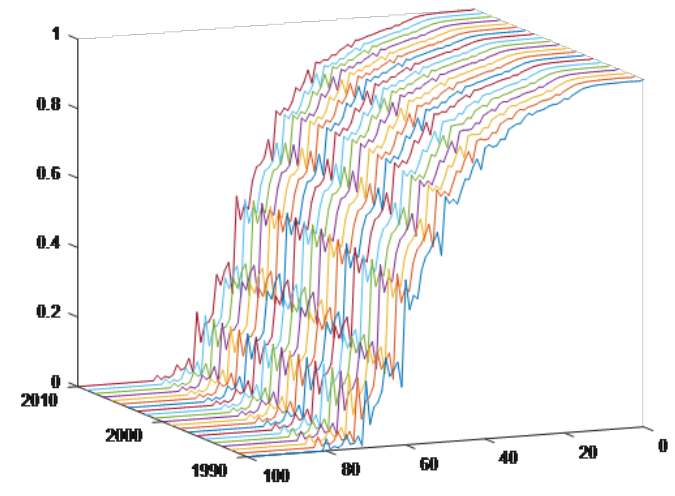

Fuente: Elaboración propia. De la ecuación (9) y datos de la Secretaría de Salud e INEGI. 
Ahora, se utilizaron las ecuaciones (7), (8), (9) y (10) y se hizo la proyección hasta el año 2040 considerando información disponible al 2010, del valor de la tasa central de la mortalidad en hombres y se determinó el valor del bono en función del año t y la edad x. El resultado obtenido del valor del bono se gráfica en 10 .

Gráfica 10. Valor estimado del Bono que considera una tasa de mortandad de hombres a la edad $\mathrm{x}$ proyectadas al 2040

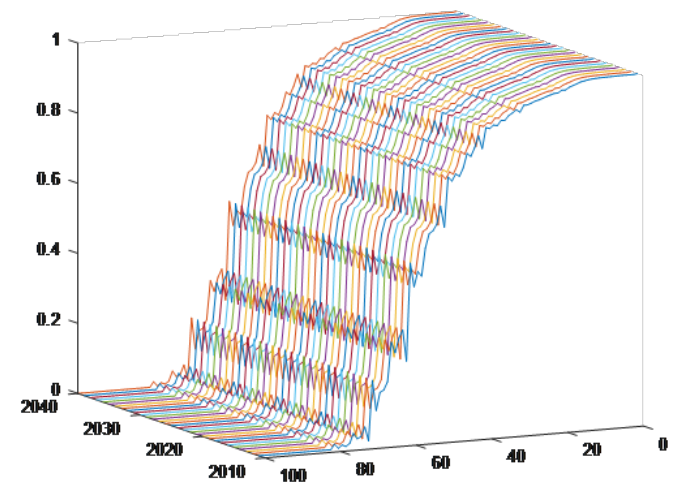

Fuente: Elaboración propia. De la ecuación (9) y (10) y datos de la Secretaría de Salud e INEGI.

Dentro de los distintos instrumentos financieros de deuda enfocado al financiamiento del sistema de pensiones mexicano se ha considerado el bono de longevidad, en este se busca contrarrestar las pérdidas probables en las que podría incurrir una institución de seguros como consecuencia de la extensión de vida esperada de los asegurados y por otro parte bajar la probabilidad de incumplimiento del corporativo ante ellos y brindándoles mayor certidumbre; así coadyuvar en dicho fondo al tener acceso al mercado de capitales y proporcione al retirado un mejor bienestar social. El bono de longevidad puede pagar dividendos a los asegurados en el futuro o bien el asegurado podría tener un pago único en el momento de su jubilación. En este documento se ha calculado el valor del bono de longevidad de un solo pago y para el período de 1990 al 2010, gráficas 9 y 15 respectivamente, y además el valor de éste para la tasa de mortandad de hombres y mujeres proyectada al 2040 para asegurados de la población mexicana y cuya evolución del valor del bono para la edad de $\mathrm{x}=0$, $1,2, \ldots, 100$ se presenta en la gráfica 10 obtenida para hombres y más adelante en la gráfica 16 obtenida para mujeres. Así las empresas tendrían la capacidad de administrar sus riesgos. 
408 Nueva Época REMEF (The Mexican Journal of Economics and Finance)

\subsection{Parámetros y el valor del Bono para mujeres}

A continuación se presentan en el cuadro 7,8 y 9 de las estimaciones de los parámetros $a_{x}, b_{x}$, y $k_{t}$, del modelo de Lee Carter con base en los datos del cálculo de la tasa de mortalidad, $m(x, t)$, para mujeres.

Cuadro 7. Parámetro $\hat{a}(x)$ a la edad x.

\begin{tabular}{|cc|cc|cc|cc|cc|}
\hline$x$ & $\hat{a}(x)$ & $x$ & $\hat{a}(x)$ & $x$ & $\hat{a}(x)$ & $x$ & $\hat{a}(x)$ & $x$ & $\hat{a}(x)$ \\
\hline 0 & -4.0866 & & & & & & & & \\
1 & -6.3136 & 21 & -7.3871 & 41 & -6.0849 & 61 & -4.0525 & 81 & -2.217 \\
2 & -7.0584 & 22 & -7.4309 & 42 & -6.3169 & 62 & -4.2956 & 82 & -2.511 \\
3 & -7.5484 & 23 & -7.3889 & 43 & -6.1166 & 63 & -4.2555 & 83 & -2.364 \\
4 & -7.8773 & 24 & -7.37 & 44 & -5.9777 & 64 & -4.1498 & 84 & -2.3486 \\
5 & -8.0086 & 25 & -7.3906 & 45 & -6.0728 & 65 & -4.3181 & 85 & -2.5496 \\
6 & -8.1072 & 26 & -7.2927 & 46 & -5.7884 & 66 & -3.9378 & 86 & -2.231 \\
7 & -8.172 & 27 & -7.2532 & 47 & -5.6609 & 67 & -3.8515 & 87 & -2.1221 \\
8 & -8.2549 & 28 & -7.2286 & 48 & -5.7338 & 68 & -3.9207 & 88 & -2.0429 \\
9 & -8.2457 & 29 & -7.1666 & 49 & -5.6086 & 69 & -3.6884 & 89 & -2.0543 \\
10 & -8.2956 & 30 & -7.2471 & 50 & -5.7712 & 70 & -4.1015 & 90 & -2.359 \\
11 & -8.2044 & 31 & -6.9238 & 51 & -5.0935 & 71 & -3.1208 & 91 & -1.1866 \\
12 & -8.205 & 32 & -7.0511 & 52 & -5.3137 & 72 & -3.5101 & 92 & -1.5351 \\
13 & -8.0504 & 33 & -6.9168 & 53 & -5.1415 & 73 & -3.331 & 93 & -1.4131 \\
14 & -7.9612 & 34 & -6.8612 & 54 & -5.1174 & 74 & -3.2884 & 94 & -1.3812 \\
15 & -7.8115 & 35 & -6.8146 & 55 & -5.1402 & 75 & -3.5055 & 95 & -1.6257 \\
16 & -7.6544 & 36 & -6.7228 & 56 & -4.9341 & 76 & -3.1413 & 96 & -1.5407 \\
17 & -7.5969 & 37 & -6.5588 & 57 & -4.7184 & 77 & -2.9458 & 97 & -1.3892 \\
18 & -7.5792 & 38 & -6.6265 & 58 & -4.7995 & 78 & -3.1231 & 98 & -1.7563 \\
19 & -7.4579 & 39 & -6.497 & 59 & -4.641 & 79 & -2.8175 & 99 & -1.9215 \\
20 & -7.5291 & 40 & -6.6209 & 60 & -4.9074 & 80 & -3.2612 & 100 & -0.9644 \\
\hline
\end{tabular}

Fuente: Elaboración propia. Datos de la Secretaría de Salud e INEGI.

Cuadro 8. Parámetro $\hat{b}(x)$ a la edad $\mathrm{x}$.

\begin{tabular}{|cc|cc|cc|cc|cc|}
\hline$x$ & $\hat{b}(x)$ & $x$ & $\hat{b}(x)$ & $x$ & $\hat{b}(x)$ & $x$ & $\hat{b}(x)$ & $x$ & $\hat{b}(x)$ \\
\hline 0 & 0.0227 & & & & & & & & \\
1 & 0.0414 & 21 & 0.0103 & 41 & 0.0156 & 61 & 0.0113 & 81 & 0.0018 \\
2 & 0.0362 & 22 & 0.0123 & 42 & 0.0188 & 62 & 0.0111 & 82 & 0.0037 \\
3 & 0.0292 & 23 & 0.0109 & 43 & 0.0105 & 63 & 0.0116 & 83 & 0.0011 \\
4 & 0.0246 & 24 & 0.012 & 44 & 0.0114 & 64 & 0.0108 & 84 & 0.0038 \\
5 & 0.0261 & 25 & 0.0102 & 45 & 0.0108 & 65 & 0.0034 & 85 & -0.0027 \\
\hline
\end{tabular}




\begin{tabular}{|cc|cc|cc|cc|cc|}
\hline$x$ & $\hat{b}(x)$ & $x$ & $\hat{b}(x)$ & $x$ & $\hat{b}(x)$ & $x$ & $\hat{b}(x)$ & $x$ & $\hat{b}(x)$ \\
\hline 6 & 0.0218 & 26 & 0.0105 & 46 & 0.0118 & 66 & 0.0058 & 86 & 0.0018 \\
7 & 0.0226 & 27 & 0.0139 & 47 & 0.0118 & 67 & 0.0056 & 87 & 0.0028 \\
8 & 0.0191 & 28 & 0.0117 & 48 & 0.009 & 68 & 0.0049 & 88 & -0.0025 \\
9 & 0.0181 & 29 & 0.0088 & 49 & 0.0086 & 69 & 0.0077 & 89 & -0.0072 \\
10 & 0.0175 & 30 & 0.0108 & 50 & 0.0052 & 70 & -0.0024 & 90 & -0.0133 \\
11 & 0.0123 & 31 & 0.0137 & 51 & 0.0084 & 71 & 0.0073 & 91 & 0.0086 \\
12 & 0.012 & 32 & 0.0164 & 52 & 0.0101 & 72 & 0.0059 & 92 & 0.0032 \\
13 & 0.0058 & 33 & 0.0149 & 53 & 0.0052 & 73 & 0.0063 & 93 & 0.003 \\
14 & 0.0066 & 34 & 0.0144 & 54 & 0.007 & 74 & 0.0061 & 94 & -0.0009 \\
15 & 0.0043 & 35 & 0.0167 & 55 & 0.004 & 75 & -0.0027 & 95 & 0.0056 \\
16 & 0.0051 & 36 & 0.0201 & 56 & 0.0083 & 76 & -0.0012 & 96 & 0.0003 \\
17 & 0.0078 & 37 & 0.0199 & 57 & 0.0071 & 77 & -0.0003 & 97 & 0.0076 \\
18 & 0.0102 & 38 & 0.0202 & 58 & 0.0062 & 78 & -0.0011 & 98 & 0.0019 \\
19 & 0.0103 & 39 & 0.0153 & 59 & 0.0081 & 79 & -0.0033 & 99 & 0.0139 \\
20 & 0.0086 & 40 & 0.0181 & 60 & 0.0012 & 80 & -0.0086 & 100 & 0.0676 \\
\hline
\end{tabular}

Fuente: Elaboración propia. Datos de la Secretaría de Salud e INEGI.

Cuadro 9. Parámetro $\hat{k}(t)$ al tiempo t.

\begin{tabular}{|ccc|ccc|}
\hline$t$ & fecha & $\hat{k}(t)$ & $t$ & fecha & $\hat{k}(t)$ \\
\hline 0 & 1990 & 17.7628 & & & \\
1 & 1991 & 14.2304 & 11 & 2001 & -6.4524 \\
2 & 1992 & 11.4323 & 12 & 2002 & -5.7749 \\
3 & 1993 & 10.7476 & 13 & 2003 & -6.5429 \\
4 & 1994 & 8.6362 & 14 & 2004 & -7.4872 \\
5 & 1995 & 8.1976 & 15 & 2005 & -6.6322 \\
6 & 1996 & 0.9305 & 16 & 2006 & -6.8945 \\
7 & 1997 & 0.1184 & 17 & 2007 & -6.1929 \\
8 & 1998 & -1.0879 & 18 & 2008 & -6.803 \\
9 & 1999 & -3.7957 & 19 & 2009 & -3.2149 \\
10 & 2000 & -5.9086 & 20 & 2010 & -5.2687 \\
\hline
\end{tabular}

Fuente: Elaboración propia.

Tomando los valores estimados de $k_{t}$ para mujeres y considerando un autoregresivo de orden uno, $\operatorname{AR}(1)$, es decir tomando la ecuación (7), se tiene que

$$
\hat{k}_{t}=0,979454 \hat{k}_{t-1}
$$

Por lo que de manera análoga que en hombres, la predicción para los años $t_{n}+s, s=1,2, \ldots, 30$ posteriores al último $t_{n}$, se realiza sustituyendo en el modelo de Lee-Carter la predicción $\hat{k}_{t_{n+s}}$ obtenida a partir de la serie temporal ajustada. Con la ecuación (11) se calcula el valor del Bono cupón cero. Se puede observar que para valores positivos del estimador $\hat{k}_{t}$ impacta en el valor del bono aumentado su valor siempre que el estimador $\hat{b}_{x}>0$ y viceversa. 
Gráfica 11. La descripción de $k_{t}$ y su auto-regresivo de orden uno

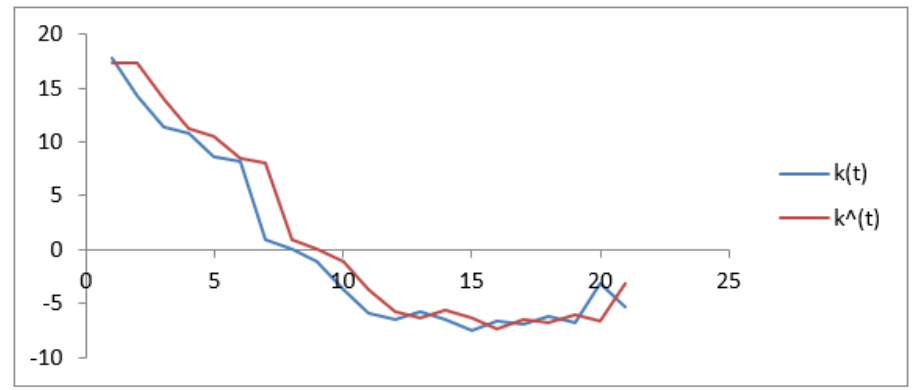

Fuente: Elaboración propia.

En el cuadro 10 se presenta el estadístico de la bondad de ajuste, $R^{2}$, que indica el porcentaje que es capaz de describir el modelo del comportamiento de los datos observados de la tasa de mortandad, $m(x, t)$, para mujeres y los estimados $\hat{m}(x, t)$ con el modelo de Lee Carter.

Cuadro 10. Bondad de ajuste de la tasa de mortandad $m(x, t)$ y $\hat{m}(x, t)$.

\begin{tabular}{|ccc|ccc|c|}
\hline & & & & & & $R^{2}(t)$ \\
$t$ & Año & $R^{2}(t)$ & $t$ & Año & $R^{2}(t)$ & Promedio \\
\hline 0 & 1990 & $99.48 \%$ & & & & $99.77 \%$ \\
1 & 1991 & $99.82 \%$ & 11 & 2001 & $99.86 \%$ & \\
2 & 1992 & $99.78 \%$ & 12 & 2002 & $99.84 \%$ & \\
3 & 1993 & $99.77 \%$ & 13 & 2003 & $99.87 \%$ & \\
4 & 1994 & $99.70 \%$ & 14 & 2004 & $99.84 \%$ & \\
5 & 1995 & $99.73 \%$ & 15 & 2005 & $99.89 \%$ & \\
6 & 1996 & $99.56 \%$ & 16 & 2006 & $99.78 \%$ & \\
7 & 1997 & $99.70 \%$ & 17 & 2007 & $99.84 \%$ & \\
8 & 1998 & $99.74 \%$ & 18 & 2008 & $99.82 \%$ & \\
9 & 1999 & $99.87 \%$ & 19 & 2009 & $99.71 \%$ & \\
10 & 2000 & $99.91 \%$ & 20 & 2010 & $99.64 \%$ & \\
\hline
\end{tabular}

Fuente: Elaboración propia.

Se grafica en 11 el logaritmo natural de la tasas de mortalidad de datos históricos en el año 2010 y los estimados con el modelo de Lee Carter para el año 2010 con objeto de visualizar el ajuste en un año y en el que se observa del cuadro 10 que tiene una bondad de ajuste de $R^{2}=99,64 \%$, es decir es el porcentaje que es capaz de explicar o describir de los datos observados de la tasa de mortandad. 
Gráfica 12. Logaritmo natural de la Tasa de mortalidad del histórico y estimado para hombres.

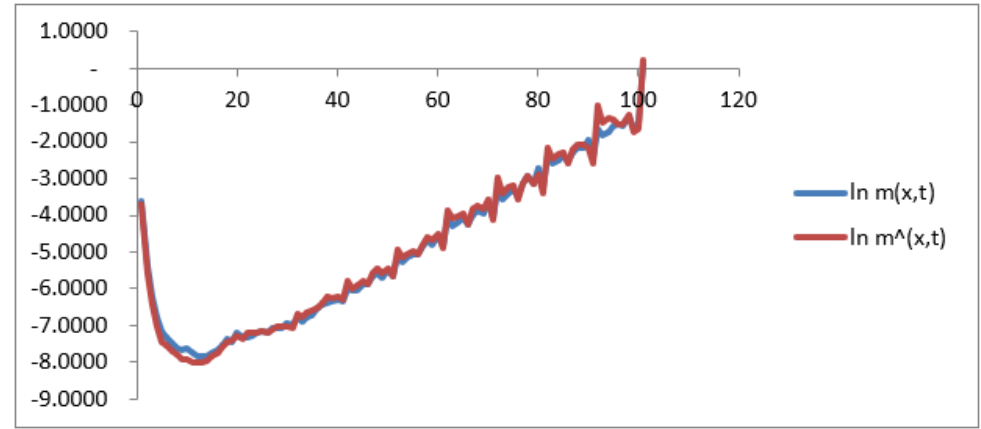

Fuente: Elaboración propia. Datos de la Secretaría de Salud e INEGI.

Se grafica el logaritmo natural de la tasas de mortalidad estimada, $\ln \hat{m}(x, t)$, en el periodo de 1990 al año 2010 de la ecuación (5) en la que se estimaron los parámetros $a_{x}, b_{x}$, y $k_{t}$ y cuyos valores se encuentran en el cuadro 1,2 , y 3 para mujeres.

Gráfica 13. El logaritmo natural de la tasas de mortalidad estimada, $\ln \hat{m}(x, t)$

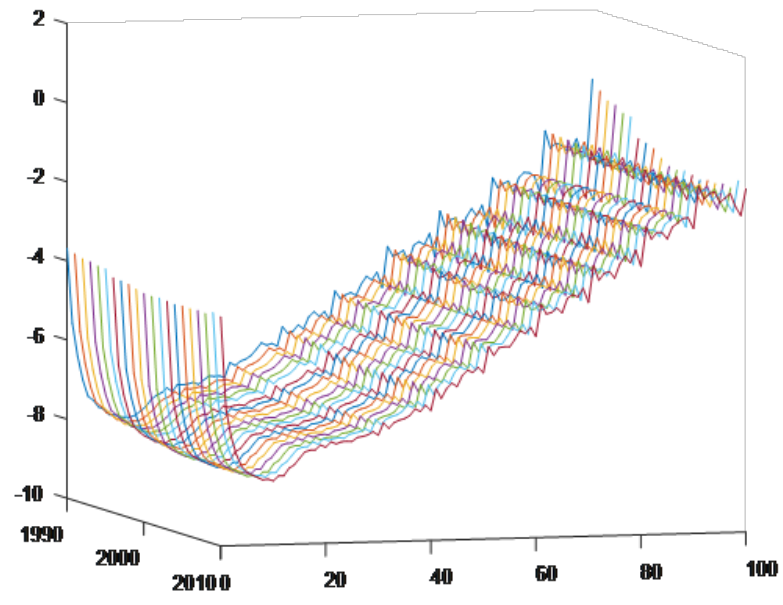

Fuente: Elaboración propia. Datos de la Secretaría de Salud e INEGI. 
412 Nueva Época REMEF (The Mexican Journal of Economics and Finance)

Gráfica 14. Dinámica de la tasa de mortalidad, $\hat{m}(x, t)$, de la población de mujeres a la edad $\mathrm{x}$, en el año calendario t.

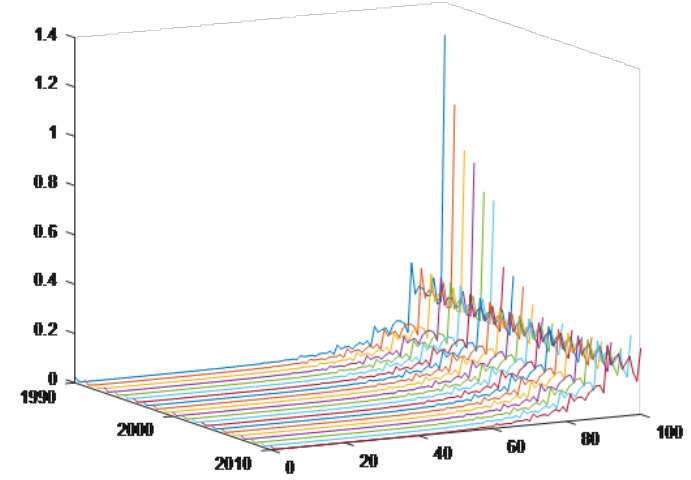

Fuente: Elaboración propia.

\subsubsection{El cálculo del Bono a través de $\hat{m}(x, t)$}

Considerando la ecuación (8), (9) y (10) se calculó el valor del bono con la tasa $\hat{m}(t, x)$ estimada para mujeres, por lo que la gráfica 15 indica el valor del bono en función del año $t \mathrm{y}$ la edad $\mathrm{x}$.

Gráfica 15. Valor estimado del Bono que considera una tasa de mortandad de mujeres a la edad $\mathrm{x}$

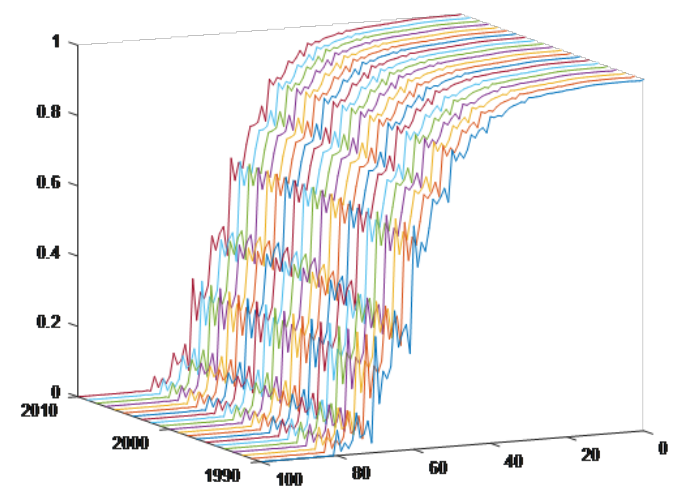

Fuente: Elaboración propia. De la ecuación (9) y datos de la Secretaría de Salud e INEGI. 
Ahora, se utilizaron las ecuaciones (7), (8), (9) y (11) y se hizo una proyección al año 2040 considerando información disponible al 2010, del valor de la tasa central de la mortalidad en mujeres y se determinó el valor del bono en función del año t y la edad x. El resultado obtenido del valor del bono se gráfica en 16 .

Gráfica 16. Valor estimado del Bono que considera una tasa de mortandad de mujeres a la edad x en el periodo de 2011 al 2040

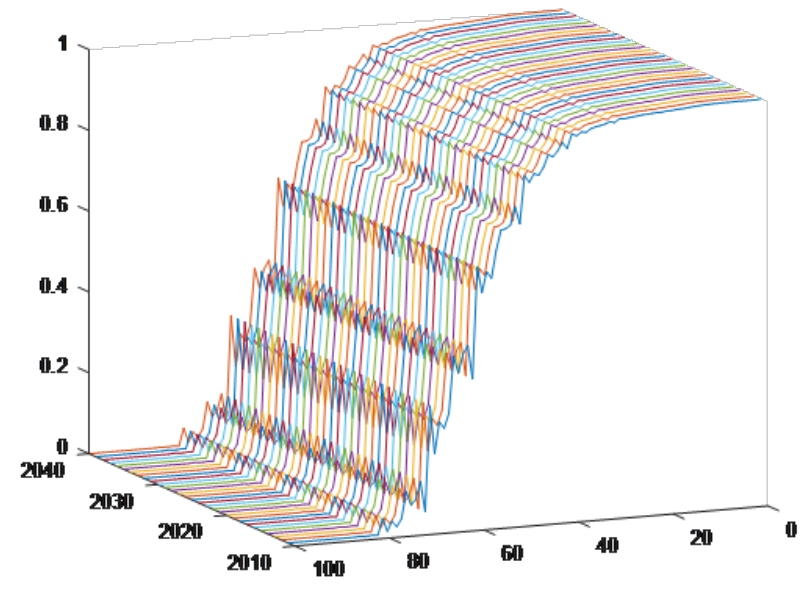

Fuente: Elaboración propia. De la ecuación (9) y (3) y datos de la Secretaría de Salud e INEGI.

\section{Conclusiones}

Se observa de los cuadros 1, 2 y graficas 1 y 3 que la mortandad de hombres y mujeres en el periodo del año 1990 al 2010 ha ido decreciendo y su esperanza de vida se está incrementando lo que implica un riesgo de longevidad en las aseguradoras. El utilizar la metodología de Lee Carter en la descripción de las tasas centrales de mortalidad de ambos géneros ha permitido obtener una bondad de ajuste en dicha tasa de hombres bastante adecuada, es decir permite explicar las observaciones en un $99.74 \%$ mientras que en el caso del género opuesto el modelo explica el índice de mortalidad en un $99.77 \%$.

Dentro de los diversos instrumentos financieros de deuda enfocado al financiamiento del sistema de pensiones mexicano se consideró el bono de longevidad, en el cual se busca contrarrestar las pérdidas probables en las que podría incurrir una institución de seguros como consecuencia de la extensión de la vida esperada de los asegurados y por otra parte bajar la probabilidad de incumplimiento del corporativo ante ellos y brindándoles mayor certidumbre; así coadyuvar en dicho fondo al disponer del acceso al mercado de capitales y proporcione al retirado un mejor bienestar social. El bono de longevidad brinda al asegurado en 
el futuro y frente a cambios demográficos, es decir incremento en la esperanza de vida y caída en la tasa de natalidad, obtener un solo pago en el momento de su jubilación, al cumplir los 65 años. En el documento se ha calculado el valor del bono de longevidad de pago único para el período de 1990 al 2010, gráficas 9 y 15 respectivamente, y además el valor de éste para la tasa de mortandad de hombres y mujeres proyectada al 2040 y cuya evolución del valor del bono a la edad de $\mathrm{x}=0,1,2, \ldots, 100$ y que se presenta en la gráfica 10 obtenida para hombres y en la gráfica 16 de mujeres. Esto daría información a las empresas enfocadas a este tipo de medios de negociación al saber los valores esperados de los bonos y que están relacionados con el índice de mortandad de la población lo que los conduciría a la toma de decisiones de manera más robusta y por otro lado los asegurados contar con un plan de ahorro que le dé una digna calidad de vida.

El valor del bono cupón cero fue modelado a través de una ecuación que incorpora la tasa de mortalidad estimada y al tiempo t, de cada género de la población y que permite facilitar el diseño de posiciones específicas de cobertura, con una doble perspectiva: una dimensión relacionada con el seguimiento de la cohorte y otra con la fecha de vencimiento. Además, en dicha valuación se utilizó un auto-regresivo de orden uno que está en función del tiempo. Por lo tanto, el objetivo final es que las personas dispongan de un plan de pensiones que les permita tengan ingresos suficientes para financiar su vejez y por otro lado brindar a los corporativos instrumentos financieros como los bonos de longevidad a fin de tener decisiones más robustas de financiamiento.

Dentro de las líneas de investigación se considera realizar una valuación de bonos con cupón para hombres y mujeres en la que se considere el índice de mortandad. Así como considerar la administración de la cartera del sistema de pensiones del corporativo con la capacidad de tomar decisiones más robustas que minimicen los riesgos de la empresa o institución. También realizar una extensión del documento en otro en el que se incluyan modelos que utilizan por ejemplo alguna transformación con objeto de realizar un análisis del comportamiento de la tasa de mortandad.

\section{Bibliografía}

Bauer, D. (2006), "An arbitrage-free family of longevity bonds", Working paper, University of Ulm.

Bauer, Daniel. Ru Jochen. (2006). Pricing Longevity Bonds using Implied Survival Probabilities. Working Paper, Ulm University and Institut fr Finanz-Aktuarwissenschaften. Available at: www.mortalityrisk.org

Blake, D., and Burrows, W. (2001), "Survivor bonds: Helping to hedge mortality risk", The Journal of Risk and Insurance, 68: 339-348.

Blake, D, Cairns, A. J.G., and Dowd, K. (2006), "Living with mortality: Longevity bonds and other mortality-linked securities". British Actuarial Journal, 12: 153-228.

Bongaarts J. (2005). "Long-range trends in adult mortality: Models and projection methods". Demography, 42, 1;23-49. 
Revista Mexicana de Economía y Finanzas, Vol. 13 No. 3, (2018), pp. 387-417

Bravo J. M. y Díaz G. J. (2014), "¿La longevidad es un riesgo asegurable? Cubriendo lo Incubrible". Vocales del Foro de Expertos del Instituto BBVA de Pensiones. Madrid. Informe 09-2014.

Brouhns, N., Denuit, M., y Vermunt, J. (2002). A Poisson log-bilinear regression approach to the construction of projected lifetables. Insurance: Mathematics Economics, 31(3):373-393.

Cairns, A.J.G., Blake, D., Dowd, K., Coughlan, G.D., Epstein, D., Ong, A., and Balevich, I. (2007), "A quantitative comparison of stochastic mortality models using data from England Wales and the United States", Working paper, Heriot-Watt University, and Pensions Institute Discussion Paper.

Cairns A.J.G., Blake D., Dowd, K., (2008a), "Modelling and Management of Mortality Risk: A Review", Pensions Institute Discussion Paper. PI-0814.

Camara de Diputados, H. Congreso de la Unión (2004). El Sistema de Pensiones (Afores) en México, 1997-2003. CEFP/008/2004.

CONAPO. Indicadores Socio demográficos. URL: http://www. conapo.gob.mx/es/CONAPO/Proyecciones_ Datos

Currie, I.D., Durban, M. And Eilers, P.H.C. (2004), "Smoothing and forecasting mortality rates", Statistical Modelling, 4: 279-284.

Currie, I.D. (2006). Smoothing and forecasting mortality rates with p-splines.

Groome, W Todd, Blancher Nicolas y Ramlongan. (Septiembre 2006), "Tercera edad y mercados financieros: El gobierno como gestor de riesgos". Finanzas Desarrollo, IMF.

INEGI, Instituto Nacional de Estadística y Geografía, URL: http://www.beta.inegi.org. $\mathrm{mx} /$ proyectos/registros/vitales/mortalidad/

Liao HH, Yang SS, Huang I-Hsing (2007), "The Design of Securitazation for Longevity Risk: Pricing under Stochastic Mortality Model with Tranche Technique". 11th APRIA Conference Taipei.

Lee, R.D., and Carter, L.R. (1992), "Modeling and Forecasting U.S. Mortality", Journal of the American Statistical Association, 87(419): 659-675.

Leppisaari, M. (2008). Managing Longevity Risk with Longevity Bonds. Helsinky.

Lin, Y. y Cox, S. (2005). Securitization of Mortality Risks in Life Annuities. The Journal of Risk and Insurance, 72: 227-252.

Levantesi Sussana, Menzietti Massimiliano y Torri Tiziana (2008). Longevity Bond Pricing Models: An Application to the Italian Annuity Market and Pension Schemes.

Martínez-Preece, M. R. y Venegas-Martínez (2014). Análisis del riesgo de mercado de los fondos de pensiones en México: un enfoque con modelos autorregresivos (2004-2010). Revista Contaduría y Administración. Vol. 59, No. 3,pp.165-195.

Miltersen, K.R., and Persson, S.A. (2005), "Is mortality dead? Stochastic forward force of mortality determined by no arbitrage" Working paper, University of Bergen.

Maurillo-López, S. y Venegas-Martínez (2011). Cobertura de los sistemas de pensiones y factores asociados al acceso a una pensión de jubilación en México. Papeles de Población. Vol. 17, No. 67, pp. 209-250.

OECD Publishing (2005). Financial Market Trends: Ageing and Pension System Reform: Implications for Financial Markets and Economic Policies, Business Economics. 74 pp.

Renshaw, A. y Haberman, S. (2003a, b). Lee-Carter mortality forecasting with age specific enhancement. Insurance: Mathematics Economics, 33(2):255-272.

Renshaw, A.E. and Haberman, S. (2003c), "Lee-Carter mortality forecasting with age specific enhancement". Insurance: Mathematics and Economics 33, 255-272.

Renshaw, A.E., and Haberman, S. (2006), "A cohort-based extensión to the Leer-Carter model for mortality reduction factors", Insurance: Mathematics and Economics, 38: 556-570.

Ruiz F. (2016). "Bono de longevidad: Instrumento financiero con un gran potencial futuro". Revista de investigación en modelos financieros. Año 5. Vol. 1. 77-108.

Shores, T. (2004). Applied Linear Algebra and Matrix Analysis. Heildelberg: Springer Verlag.

Tsay R. S. (2010). Analysis of Financial Time Series. John Wiley Sons, Inc. Publication.

Vásquez Colmenares G. P. (2012), "Nueva seguridad social y la crisis de las pensiones". ECONOMÍA. UNAM. Vol. 10. Número. 28. 
416 Nueva Época REMEF (The Mexican Journal of Economics and Finance)

Wang, Jennifer L., Yang, Sharon S. (2002), "A universal framework for pricing financial and insurance risks." ASTIN Bulletin, Vol. 32. Num. 2, 213-234.

Wang, Jennifer L., Yang, Sharon S. (2008), "Pricing and Implementation of Longevity Bonds in Taiwan." Asia-Pacific Journal of Risk and Insurance, 3:155-171.

\section{Apéndice A1}

A1. Estimación de parámetros utilizando descomposición en valores singulares, DVS

A continuación se presenta la descripción para llevar a cabo la estimación de los parámetros del modelo de Lee C. a través de la descomposición en valores singulares.

La función a minimizar está dada por

$$
D(\bar{a}, \bar{b}, \bar{k})=\sum_{x, t}\left(\log \left(m_{x, t}\right)-\left(a_{x}+b_{x} k_{t}\right)\right)^{2}
$$

donde los parámetros $\overline{a_{x}}, \overline{b_{x}} \in \mathbb{R}^{n_{a}}$ y $\bar{k} \in \mathbb{R}^{n_{c}}$, y sea $M=\left[\log \left(m_{x, t}\right)\right] \in$ $\mathbb{R}^{n_{a} \times n_{c}}$ con $\mathbb{I}=\left(\begin{array}{c}1 \\ \vdots \\ 1\end{array}\right), \quad \mathbb{I}^{t} \in \mathbb{R}^{n_{c}}$. Así, $D(\bar{a}, \bar{b}, \bar{k})$ es la norma de $\log \left(m_{x, t}\right)$ $\left(a_{x}+b_{x} k_{t}\right)$ la cual queda expresada como

$$
D(\bar{a}, \bar{b}, \bar{k})=\left\|M-a_{x} \mathbb{I}^{\prime}-b_{x} k_{t}^{\prime}\right\|_{2}^{2}
$$

en la que $\|A\|_{2}$ es la norma euclídea en $\mathbb{R}^{n_{a} \times n_{c}}$ y a esta norma dada por $\|A\|_{2}=\sqrt{\sum_{i} \sum_{j}\left|A_{i, j}\right|^{2}}=\sqrt{\operatorname{trazaAA}}$ se le denomina la norma de Frobenius en $\mathbb{F}^{m \times n}$ espacio vectorial para matrices o norma de Hilbert-Schmidt. Note que en $a_{x} \mathbb{I}^{\prime}$ está uno de los parámetros y cuyas estimaciones estarán representados por $\widehat{\widehat{a}} \mathbb{I}^{\prime}$ en la que $\mathrm{x}=0,1,2, \ldots, 100$ años de edad de la persona asegurada por un corporativo o Institución y junto a $\widehat{\bar{b}}$ el parámetro $\widehat{\bar{k}}_{t}$ en la que $\mathrm{t}=1,2,3$, ... el tiempo en años como se muestra en la ecuación (2), en particular $\mathrm{t}=1,2,3$, ...21, esto es de 1990 a 2010; posteriormente considerar la proyección al 2040. Se verifica que el producto de las matrices $b_{x} k_{t}{ }^{\prime}$ son de $101 \times 21$ y de $21 \times 1$ respectivamente y $a_{x} \mathbb{I}^{\prime}$ son de $101 \times 21$ y de $21 \times 1$ analogamente. Así mismo, se puede observar que en este caso la matriz $M=\left[\log \left(m_{x, t}\right)\right]$ representa la tasa de mortandad de los asegurados en la que $\mathrm{x}=0,1,2, \ldots, 100$ años de edad $\mathrm{y}$ en el año $\mathrm{t}=1,2,3, \ldots 21$.

Considerando la condición de primer orden para minimizar, se tiene que $\partial D / \partial \bar{a}=0$ y $\sum_{\tau} k_{\tau}=0, \sum_{x} b_{x}=1$, así se tiene como solución el vector dado por los promedios de la matriz $\mathrm{M}$, con $\widehat{\bar{a}}=\frac{1}{n_{c}} M \mathbb{I}$. Al sustituir la solución $\widehat{\bar{a}}$ en la ecuación (1), se tiene que minimizar con respecto a los parámetros $(\bar{b}, \bar{k})$ en la función

$$
D(\bar{b}, \bar{k})=\left\|M-\hat{a} \mathbb{I}^{\prime}-\hat{b} k_{t}^{\prime}\right\|_{2}^{2}
$$


Entonces, minimizar la ecuación (2) resulta un caso particular de un problema general de aproximar una matriz $\mathrm{H}$ por otra matriz con rango igual o menor, Y, es decir determinar el estimador $\hat{Y}$ tal que

$$
\hat{Y}=\operatorname{argmin}_{\text {rango }(X) \leq r}\|H-Y\|_{2}
$$

con $r=\operatorname{rango}(H)$ y por lo tanto se determina la solución $\hat{Y}$ a través de la descomposición singular de la matriz H. Las columnas de la matriz de interés son linealmente dependientes.

Considerando el teorema de descomposición singular (vea Shores (2004)), se tiene que la solución $\hat{Y}$ acorde a Eckart Young (1936), está dada por

$$
\hat{Y}=\sum_{j=1}^{h} \sigma_{j} u_{j} v_{j}^{\prime}
$$

En la que $\sigma_{j}, \mathrm{j}=1, \ldots, \mathrm{h}$, son los valores singulares de la matriz A y a la matriz $\sigma_{j} u_{j} v_{j}^{\prime}$ se le denomina el i-ésimo modo de A. Ahora bien, se tiene que rango $(H)=h<\operatorname{mín}(m, n)$. Entonces, $H=M-\widehat{\bar{a}} \mathbb{I}$ ' considerando que $n_{a}=$ $m<n=n_{c}$. Por lo tanto,

$$
M-\widehat{\bar{a}} \mathbb{I}^{\prime}=\sum_{j=1}^{h} \sigma_{j} u_{j} v_{j}^{\prime}
$$

Lee C. (1992) utilizó una aproximación de rango $\mathrm{h}=1$ y de ahí que $\widehat{\bar{b}}=\sqrt{\sigma_{1}} u_{1}$ y $\widehat{\bar{k}}=\sqrt{\sigma_{1}} v_{1}$. Por lo tanto, se tiene la siguiente aproximación

$$
M \approx \widehat{\bar{a}} \mathbb{I}^{\prime}+\widehat{\bar{b}} \widehat{\bar{a}}^{\prime}
$$

Para mayor profundidad en el tema, vea Koissi Shapiro (2008) y Brouhns et. al. (2002). 
418 Nueva Época REMEF (The Mexican Journal of Economics and Finance) 